\title{
Expression of neurofibromin 1 in colorectal cancer and cetuximab resistance
}

\author{
EUNYOUNG TAK ${ }^{1}$, MINHEE KIM ${ }^{2}$, YOUNGRA CHO ${ }^{2}$, SUEUN CHOI ${ }^{2}$, JIHUN KIM $^{3}$, \\ BUHM HAN ${ }^{4}$, HYUNG-DON KIM ${ }^{5}$, CHLOE SOO-HYUN JANG $^{4}$, JEONG EUN KIM ${ }^{5}$, \\ YONG SANG HONG ${ }^{5}$, SUN YOUNG KIM ${ }^{5 *}$ and TAE WON KIM ${ }^{5 *}$ \\ ${ }^{1}$ Department of Convergence Medicine, ${ }^{2}$ Asan Medical Institute of Convergence Science and Technology (AMIST) and \\ ${ }^{3}$ Department of Pathology, Asan Medical Center, University of Ulsan College of Medicine, Songpa, Seoul 05505; \\ ${ }^{4}$ Department of Biomedical Sciences, Seoul National University College of Medicine, Jongro, Seoul 03080; \\ ${ }^{5}$ Department of Oncology, Asan Medical Center, University of Ulsan \\ College of Medicine, Songpa, Seoul 05505, Republic of Korea
}

Received June 23, 2021; Accepted September 14, 2021

DOI: $10.3892 /$ or.2021.8226

\begin{abstract}
Neurofibromin 1 (NF1) is a tumor suppressor that has been previously reported to regulate RAS-MAPK signaling. The present study investigated the possible relationship between NF1 expression and anti-EGFR antibody (cetuximab) sensitivity in colorectal cancer cell lines. In addition, primary or metastatic colorectal cancer samples from patients treated with cetuximab were assessed for the association of cetuximab sensitivity. The quantities of the NF1 transcript, NF1-related pathway enrichment and NF1 mutation profile were measured and investigated using RNA sequencing and targeted DNA sequencing. Based on growth inhibition and colony formation assay results, cell lines were designated to be cetuximab-sensitive (NCI-H508 and Caco2) or cetuximab-resistant (KM12C and SM480). Western blotting revealed NF1 was highly expressed in cetuximab-sensitive cell lines whilst there was little expression in their cetuximab-resistant counterparts. Knocking down NFI expression using small interfering RNA in the cetuximab-sensitive cell lines enhanced the phosphorylation of MEK and ERK according to western blotting. NF1 knockdown also reduced apoptosis, as observed by the decreased number of apoptotic bodies by DAPI nuclear
\end{abstract}

Correspondence to: Professor Sun Young Kim or Professor Tae Won Kim, Department of Oncology, Asan Medical Center, University of Ulsan College of Medicine, 88 Olympic-ro 43-gil, Songpa, Seoul 05505, Republic of Korea

E-mail: sunyoungkim@amc.seoul.kr

E-mail: twkimmd@amc.seoul.kr

${ }^{*}$ Contributed equally

Key words: neurofibromin 1, cetuximab resistance, colorectal cancer staining and reduced cleavage of caspase and poly-(ADP ribose) polymerase. NF1 overexpression by transfection with GTPase-activating protein-related domain subunit rendered the cetuximab-resistant cell lines, KM12C and SW480, more susceptible to cetuximab-induced apoptosis. RNA sequencing of $111 R A S$ and $B R A F^{\mathrm{V} 600}$ wild-type tumor samples collected from cetuximab-treated patients with metastatic colorectal cancer revealed that the pre-treatment $N F 1$ expression levels were not associated with the cetuximab response. However, tumor samples obtained after cetuximab treatment displayed slightly lower NF1 transcript levels compared with those in the pre-treatment samples, suggesting that exposure to the anti-EGFR antibody may be associated with reduced NF1 expression levels. Next-generation sequencing revealed that the frequency of inactivating mutations in $N F 1$ were rare $(1.8 \%)$ in patients with colorectal cancer and were not associated with the protein expression levels of NF1 except for in a small number of cases $(0.5 \%)$, where the biallelic inactivation of NF1 was observed. To conclude, the present study showed that modification of NF1 expression can affect sensitivity to cetuximab in colorectal cancer cell lines, though a limitation exists in terms of its potential application as a biomarker for $R A S$ and $B R A F^{\mathrm{V} 600}$ wild-type tumors.

\section{Introduction}

Colorectal cancer (CRC) is estimated to be the third most common cancer and the second common cause of cancer-related mortality worldwide in 2020, accounting for $\sim 900,000$ deaths annually (1). Therapeutic strategies for metastatic colorectal cancer (mCRC) have improved over the past number of decades, which resulted in prolonged patient survival for $\leq 3$ years (2). However, an urgent demand exists for in the treatment of patients who have progressed even after treatment with cytotoxic chemotherapy and targeted agents (3). Although anti-EGFR antibodies, such as cetuximab or panitumumab, have been proven to be effective against $R A S$ oncogene wild-type $\mathrm{mCRC}$, intrinsic and acquired resistance 
has provided a major obstacle during this particular course of treatment (3). In this regard, efforts have been made to elucidate the mechanism underlying the acquisition of resistance to anti-EGFR therapy. Several signaling pathways, including RAS/RAF/MAPK, PI3K/PTEN/AKT and Janus kinase (JAK)/STAT pathways, have been revealed to be potential therapeutic targets for colorectal cancer (4). However, therapeutic approaches that were proposed for overcoming resistance to anti-EGFR therapy thus far have rarely been able to confer clinical benefits $(5,6)$. Therefore, this necessitates further investigations on the mechanism of anti-EGFR therapy resistance for the development of novel therapeutic strategies.

Neurofibromin 1 (NF1) is a protein that is 2,818 amino acids long and is a negative regulator of RAS signaling by accelerating guanosine triphosphate (GTP) hydrolysis by the RAS protein (7). In addition, NF1 is among the potential targets that have been previously implicated in mediating anti-EGFR resistance, specifically in lung cancer and CRC $(8,9)$. Profiles on somatic NF1 aberrations in solid tumors, including lung cancer, breast cancer and melanoma, have been previously established by various cancer genome sequencing projects (10-12), which enabled in-depth studies into the therapeutic implications of those aberrations (13). A number of translational studies have previously shown that gene mutations in NF1 or the levels of NF1 expression can influence the therapeutic efficacy of anti-cancer treatments, including BRAF inhibitors for melanoma, anti-EGFR treatments for lung cancer, tamoxifen for breast cancer and retinoic acids for neuroblastoma (14-16). However, little is known about the effects of differential expression NF1 levels on the therapeutic outcome in the context of anti-EGFR therapy for CRC.

In the present study, the potential association between NF1 expression and response to anti-EGFR treatment in CRC cell lines was investigated. In addition, the possible effects of manipulating NF1 expression on sensitivity to anti-EGFR treatment were explored. Subsequently, NF1 expression levels in tumor samples from patients who were treated with anti-EGFR therapy were measured, following which the incidence of NF1 mutations in the patient database (Genomic Laboratory Information System of Asan Medical Center, Seoul, South Korea) was explored after genomic profiling.

\section{Materials and methods}

Colorectal cancer cell lines. In total, four CRC cell lines, NCI-H508, Caco-2, KM12C and SW480, were obtained from Korean Cell Line Bank (Korean Cell Line Research Foundation). According to a previous study (17), NCI-H508, Caco-2 and KM12C are of the wild-type KRAS/BRAF $F^{\mathrm{v} 600}$ genotype, whilst KM12C originated from a microsatellite-high tumor harboring NF1 mutations (T676fs, F945L, L1361R). SW480 is a KRAS G12V mutant cell line. The NCI-H508 and SW480 cells were cultured in RPMI-1640 (cat. no. SH30027.01; Hyclone; Cytiva) supplemented with $10 \%$ FBS (Gibco; Thermo Fisher Scientific, Inc.) and $100 \mathrm{U} / \mathrm{ml}$ penicillin/streptomycin (Hyclone; Cytiva). The Caco-2 and KM12C cells were cultured in minimum essential medium (MEM; cat. no. LM007-07; Welgene, Inc.) supplemented with $10 \% \mathrm{FBS}$ and $100 \mathrm{U} / \mathrm{ml}$ penicillin/streptomycin. All cells were maintained at $37^{\circ} \mathrm{C}$ under $5 \% \mathrm{CO}_{2}$ in a humidified atmosphere and passaged for a maximum of 6 months. For cell lines used in the present study, the short tandem repeat (STR) was verified by the Korean Cell Line Bank; Korean Cell Line Research Foundation. All cell cultures were checked using an EZ-PCR mycoplasma detection kit (cat. no. SKU:20-700-20; Biological Industries), and all of them were free from mycoplasma contamination.

Western blotting. Cells were trypsinized, washed with ice-cold PBS and lysed with the RIPA lysis buffer [50 mM HEPES (pH 7.4), $150 \mathrm{mM} \mathrm{NaCl}, 1$ mM EDTA, 2.5 mM EGTA, 1 mM dithiothreitol and $1 \%$ Triton X-100] containing a protease and phosphatase inhibitor cocktail (Sigma-Aldrich; Merck KGaA). After lysis, the cell debris were removed by centrifugation at $20,000 \mathrm{x}$ g for $20 \mathrm{~min}$ at $4^{\circ} \mathrm{C}$. Protein concentration was determined using Bradford assay. The cellular protein samples (30 $\mu \mathrm{g}$ ) were separated by $8-15 \%$ SDS-PAGE and transferred onto nitrocellulose membranes. The membranes were blocked with 5\% non-fat dry milk in TBST $(20 \mathrm{mM}$ Tris- $\mathrm{HCl} \mathrm{pH} 7.4$, $150 \mathrm{mM} \mathrm{NaCl}$ and $0.1 \%$ Tween-20) for $1 \mathrm{~h}$ at room temperature and probed with anti-NF1 (1:1,000; cat.no. 14623; Cell Signaling Technology, Inc.), anti-MEK1/2 (1:1,000; cat. no. 9122; Cell Signaling Technology, Inc.), anti-phosphorylated (p-)-MEK1/2 (ser217/221; 1:1,000, cat. no. 9121; Cell Signaling Technology, Inc.), anti-ERK1/2 (1:1,000; cat. no. 9102; Cell Signaling Technology, Inc.), anti-p-ERK1/2 (Thr202/tyr204; 1:1,000; cat. no. 9101; Cell Signaling Technology, Inc.), anti-caspase-3 (1:1,000; cat. no. 9662; Cell Signaling Technology, Inc.), anti-cleaved (c-)-caspase-3 (Asp175; 1:1,000; cat. no. 9661; Cell Signaling Technology, Inc.), anti-PARP (1:1,000; cat. no. ab32138; Abcam), anti-c-PARP (1:1,000; cat. no. 9532S; Cell Signaling Technology, Inc.) or anti-actin (1:20,000; cat. no. A3854; Sigma-Aldrich; Merck KGaA) primary antibodies for overnight at $4^{\circ} \mathrm{C}$. After washing with TBST, the membranes were incubated with HRP-conjugated goat anti-mouse $(1: 10,000$; cat. no. 31430; Thermo Fisher Scientific, Inc.) or goat-rabbit (1:10,000; cat. no. 31460; Thermo Fisher Scientific, Inc.) secondary antibodies for $1 \mathrm{~h}$ at room temperature. The proteins were developed using the chemiluminescent (ECL) substrate (SuperSignal ${ }^{\mathrm{TM}}$ West Femto Maximum Sensitivity Substrate; cat. no. 34095; Thermo Fisher Scientific, Inc.). A detection system (LuminoGraph II; cat. no. WSE-6200; ATTO Corporation) with a controlling software (ImageSaver 6; version 2.7.2; ATTO Corporation) was used to visualize the bands. The obtained band images were quantified by densitometry analysis using the ImageJ software (v1.53a; National Institutes of Health).

Reverse transcription-quantitative PCR (RT-qPCR). Total RNA was collected from cells using the QIAzol lysis reagent (cat. no. 79306; Qiagen $\mathrm{GmbH}$ ) and the RNeasy mini kit (cat. no. 74106, Qiagen $\mathrm{GmbH}$ ) using a modified protocol. Briefly, the CRC cells $\left(2.0 \times 10^{6}\right.$ cells) were treated with $0.5 \mathrm{ml}$ QIAzol lysis buffer and were collected, mixed with $0.1 \mathrm{ml}$ chloroform and centrifuged at $16,000 \mathrm{x}$ g for $15 \mathrm{~min}$ at $4^{\circ} \mathrm{C}$. for $10 \mathrm{~min}$. The clear supernatants were separately collected, and the nucleic acids were precipitated using $70 \%$ ethanol. The precipitated total RNAs were bound on the silica column from the RNeasy mini kit components, washed twice with the RPE buffer (provided in the kit) and eluted with RNase-free 
distilled water (provided in the kit). The concentration and quality of the extracted RNA were measured using Nanodrop 2000 (Thermo Fisher Scientific, Inc.). Samples with an optical density 260/280 value $>1.8$ were used for further experiments. The cDNA was generated from the mRNA using the ReverTra Ace $^{\mathrm{TM}}$ qPCR RT Master Mix (Toyobo Life Science) by incubating at $37^{\circ} \mathrm{C}$ for $15 \mathrm{~min}$, heating at $50^{\circ} \mathrm{C}$ for $5 \mathrm{~min}$ and cooling to $4^{\circ} \mathrm{C}$. The transcripts were quantified by qPCR using the CFX Connect Real-Time PCR Detection System (Bio-Rad Laboratories, Inc.) with 5X HOT FIREPol ${ }^{\circledR}$ EvaGreen ${ }^{\circledR}$ qPCR Supermix (Solis BioDyne). The samples were first denatured at $95^{\circ} \mathrm{C}$ for $15 \mathrm{~min}$, followed by 40 cycles of denaturation at $95^{\circ} \mathrm{C}$ for $15 \mathrm{sec}$, annealing at $55-60^{\circ} \mathrm{C}$ for $15 \mathrm{sec}$ and elongation at $72^{\circ} \mathrm{C}$ for $20 \mathrm{sec}$. The primer sequences were as follows: GAPDH forward, 5'-GAGTCAACGGATTTGGTC GT-3' and reverse, 5'-TTGATTTTGGAGGGATCTCG-3' and NF1 forward, 5'-GGATCTCCAGACAAGAGCTACA-3' and reverse, 5'-CTCTCAAACCGATCAGCCAATAC-3'. The data were expressed as the fold change in the treatment groups relative to the control and normalized to $G A P D H$ levels.

Small interfering RNA (siRNA) and plasmid construct transfection. siRNAs specific for either NF1 (NF1-siRNA) or a scrambled sequence (scrambled-siRNA) were prepared and designed by Bioneer Corporation. The siRNA sequences were as follows: NF1-siRNA1 (targeting the NF1 exon 11 region) sense, 5'-CACCUUCUACAUUUCACUA-3' and antisense, 5'-UAGUGA AAUGUAGAAGGUG-3'; NF1-siRNA2 (targeting the NF1 exon 9 region) sense, 5'-CUGUGUAAAGCA AGUACUU-3' and antisense, 5'-AAGUACUUGCUUUAC ACAG-3' and scrambled-siRNA sense, 5'-UCCCAGAUAGAG ACUUCAATT-3' and anti-sense, 5'-UUGAAGUCUCUAUCU GGGATT-3'.

The negative control plasmid (pEYFP-C1; Empty) and the NF1-GTPase-activating protein related domain (GRD)-expressing plasmids (NF1-GRD) in pEYFP-C1 were kindly provided by Professor Seon-Yong Jeong, Ajou University School of Medicine (Suwon, South Korea) (18). Briefly, the NF1-GRD expressing vectors were generated by the subcloning cDNAs of NF1-GRD into the pEYFP-C1 vector (Clontech Laboratories, Inc.) using two restriction enzymes (BgIII and HindIII). The cDNAs of the GRD region of NF1 were generated by reverse transcription-PCR from total RNAs of IMR-90 human fibroblasts (purchased from ATCC; cultured in DMEM with $10 \% \mathrm{FBS}$ at $37^{\circ} \mathrm{C}$ in $5 \%$ $\mathrm{CO}_{2}$ ) using the following primers: Forward 5'-ATAGATCTA CCATGGATCTCCAGACAAGAGCTACATTTATG-3' and reverse, 5'-GTAAGCTTAACCAGTGTGTATCTGCCACAG GT-3' (18). These primer sequences correspond to the region of human (GRCh38) chr17:31,233,018-31,261,811 (NF1 accession no. NM_001042492).

The cells were transfected with siRNAs (200 nM) or vectors $(5 \mu \mathrm{g})$ using Lipofectamine ${ }^{\circledR} 3000$ reagent (Invitrogen; Thermo Fisher Scientific, Inc.) according to the manufacturer's protocol. The efficiency of siRNA-based NF1 knockdown and plasmid transfection was assessed by RT-qPCR and western blotting. To measure the effect of siRNAs or vectors on cell viability, the relative number of cells was compared between control and transfected samples. The cells were maintained for $72 \mathrm{~h}$ after siRNA or vector transfection before images of the cells were acquired at x20 magnification using an EVOS-FL automated fluorescence microscope (Thermo Fisher Scientific, Inc.) under bright-field mode. The relative number of transfected cells was counted from the image using the ImageJ software (v1.53a; National Institutes of Health, USA). The clustering cells were counted according to particle analysis protocol (https://imagej.net).

For western blotting and nuclear staining after siRNA or plasmid transfection, cells were seeded into 12 -well plates at $5 \times 10^{5}$ cells/well prior to transfection. Cetuximab $(100 \mu \mathrm{g} / \mathrm{ml})$ was added $24 \mathrm{~h}$ after transfection and cells were collected for western blotting or nuclear staining $48 \mathrm{~h}$ after cetuximab treatment at $37^{\circ} \mathrm{C}$ under $5 \% \mathrm{CO}_{2}$ in a humidified atmosphere.

Cell growth assay. For cell growth assays, cells were seeded into 24-well plates at 5,000 cells/well and cultured in the complete medium supplemented with 0,50, 100 and $200 \mu \mathrm{g} / \mathrm{ml}$ cetuximab (Merck KGaA). The full culture medium was not changed throughout the course of the experiment. The cells were fixed in $10 \%$ neutral buffered formalin for $20 \mathrm{~min}$ at room temperature and stained with $0.1 \%$ crystal violet for $20 \mathrm{~min}$ at room temperature. The dye was extracted with $10 \%$ acetic acid, and relative proliferation levels were determined according to the optical density at $595 \mathrm{~nm}$ using a Sunrise microplate reader (Tecan Group, Ltd.).

Colony formation assay. For the colony formation assays, cells were seeded at 500 cells/well into 6-well plates and then cultured in the complete medium with 0, 50, 100 and $200 \mu \mathrm{g} / \mathrm{ml}$ cetuximab at $37^{\circ} \mathrm{C}$. The full culture medium was not changed throughout the course of the experiment. After 10 days, the cells were fixed in $80 \%$ methanol for $20 \mathrm{~min}$ at room temperature and stained with a $0.2 \%$ crystal violet for $20 \mathrm{~min}$ at room temperature. The number of colonies (diameters $>200 \mu \mathrm{m}$ ) was counted using the Oxford Optronix GelCount $^{\mathrm{TM}}$ system (v1.1.2.0; Oxford Optronix).

Nuclear staining. DAPI staining assay was conducted to detect the possible occurrence of nucleus condensation in the siRNA- and plasmid-transfected cells. The cells were fixed with freshly prepared ice-cold $10 \%$ neutral buffered formalin for $10 \mathrm{~min}$ at $4^{\circ} \mathrm{C}$ and then exposed to $0.1 \%$ Triton $\mathrm{X}-100$ in PBS for $10 \mathrm{~min}$ for permeabilization. They were subsequently stained with Fluoroshield Mounting Medium with DAPI (cat. no. ab104139; Abcam) for $1 \mathrm{~min}$ at room temperature. Image acquisition at x20 magnification was performed using the EVOS FL Auto-fluorescence microscope (Thermo Fisher Scientific, Inc.). The relative number of cells was counted using ImageJ software (v1.53a; National Institutes of Health) (19).

Tumor samples. To estimate the NF1 expression levels in the cetuximab-treated samples, RNA sequencing data from the tumor samples of patients with $\mathrm{mCRC}$ who received cetuximab were used. Patients were eligible for this study if the patient participated in the mCRC biomarker discovery program of the Department of Medical Oncology of Asan Medical Center, which enrolled those with histologically proven CRC who were supposed to undergo or were undergoing chemotherapy with adjuvants or palliative treatment. Among these, the selection criteria for the present study were as follows: 
i) Patients who received cetuximab as their treatment for mCRC before March 2018; ii) those who were followed up $\geq 6$ months from the first dose of cetuximab; iii) those who had tumor tissues adequately archived, iv) patients with samples which were confirmed as the $R A S$ and $B R A F^{\mathrm{V} 600}$ wild-type by either Sanger sequencing or next-generation sequencing (NGS); v) patients with samples which were also obtained before or after $\geq$ one dose of cetuximab administration; and vi) patients with available clinical data regarding responses to cetuximab-based treatment (Tx). Among the 2,589 participants who enrolled into the biomarker discovery program from September 2011 to March 2018, 92 patients with 113 samples met the criteria aforementioned (Table I). The samples were categorized into the following four groups: i) Pre-treatment samples from patients who achieved a complete response (CR)/partial response (PR) using Response Evaluation Criteria in Solid Tumor (RECIST) v.1.1 (20) as their best response (pre-Tx CR/PR; n=60); ii) pre-treatment samples from patients with stable disease (SD)/progressive disease (PD; pre-Tx $\mathrm{SD} / \mathrm{PD} ; \mathrm{n}=16)$; iii) post-treatment samples with non-progressive disease (post-Tx nPD; $n=16$ ) if the sample was obtained before the clinical determination of disease progression; and iv) post-progression samples (post-Tx PD; $n=21$ ) if excision or biopsy had been conducted after progression was adjudged according to RECIST v1.1.

To assess the frequency of NF1 mutations, the Genomic Laboratory Information System of Asan Medical Center genomic database containing data of patients who underwent NGS testing for diagnostic purposes was screened. The clinical NGS data of 1,449 patients with CRC who underwent testing diagnostic purposes from March 2017 to May 2020 were screened. No specific exclusion criteria was applied for this screening process. Immunohistochemical staining (IHC) for NF1 was conducted for the selected samples identified from the database.

The biomarker discovery program, including RNA sequencing and IHC staining, was approved (approval no. 2011-0511) by the Institutional Review Board of Asan Medical Center and conducted in accordance with the tenets of the Declaration of Helsinki and Good Clinical Practice.

RNA sequencing and bioinformatic analyses. Formalin-fixed ( $10 \%$, for $12-18 \mathrm{~h}$ at a room temperature) and paraffin-embedded tissues were used for transcriptomic analysis. Macro-dissection was performed from a tumor portion of an unstained $6-\mu \mathrm{m}$-thick slide and RNA was extracted using the RNeasy FFPE kit (cat. no. 7350; Qiagen $\mathrm{GmbH}$ ) according to the manufacturer's protocol. A cDNA library was constructed using the TruSeq RNA Access Library Prep Kit (cat. no. RS-301-2002; Illumina, Inc.). Briefly, mRNA was purified from total RNA using poly A selection and then cleaved and converted into double-stranded cDNA fragments using random primers. The library was prepared by the random fragmentation of cDNA samples followed by $5^{\prime}$ and $3^{\prime}$ adaptor ligation. Adaptor-ligated fragments were PCR-amplified and gel-purified before their quality was assessed with a 2100 Bioanalyzer (Agilent Technologies, Inc.) using an Agilent DNA 1000 Kit (cat. no. G2938-90015; Agilent Technologies, Inc.) (21). Samples that passed the library quality assurance process (concentration $>5 \mathrm{nM}$ and
Table I. Characteristics of the colorectal tumor samples collected from the cetuximab-treated patients with the RAS and BRAF ${ }^{\mathrm{V} 600}$ wild-type who underwent RNA-sequencing testing.

\begin{tabular}{|c|c|}
\hline Category & Values $^{\mathrm{a}}$ \\
\hline Age, years & $58(22-80)^{\mathrm{b}}$ \\
\hline \multicolumn{2}{|l|}{ Sex } \\
\hline Male & $57(62 \%)$ \\
\hline Female & $35(38 \%)$ \\
\hline \multicolumn{2}{|l|}{ Lines of treatment } \\
\hline 1 & $75(82 \%)$ \\
\hline$\geq 2$ & $17(18 \%)$ \\
\hline \multicolumn{2}{|l|}{ Regimen } \\
\hline Cetuximab & $5(5 \%)$ \\
\hline Cetuximab + irinotecan & $12(13 \%)$ \\
\hline Cetuximab + FOLFIRI & $66(72 \%)$ \\
\hline Cetuximab + FOLFOX & $9(10 \%)$ \\
\hline \multicolumn{2}{|l|}{ Primary site } \\
\hline Right & $19(21 \%)$ \\
\hline Left & $73(79 \%)$ \\
\hline \multicolumn{2}{|l|}{$\begin{array}{l}\text { Test for RAS and } \mathrm{BRAF}^{\mathrm{V} 600} \\
\text { mutations status }\end{array}$} \\
\hline Sanger sequencing & $15(16 \%)$ \\
\hline Next-generation sequencing & $77(84 \%)$ \\
\hline \multicolumn{2}{|l|}{ Initial stage } \\
\hline Stage I-III & $10(11 \%)$ \\
\hline Stage IV & $82(89 \%)$ \\
\hline \multicolumn{2}{|l|}{ MSI status } \\
\hline MSI-H & $1(1 \%)$ \\
\hline MSI-L & $3(3 \%)$ \\
\hline MSS or pMMR & $86(93 \%)$ \\
\hline Not tested & $2(2 \%)$ \\
\hline
\end{tabular}

Progression-free survival

First line, months

$\geq$ Second line or more, months

$13.48(12.66-14.66)^{\mathrm{c}}$

$6.46(1.57-9.41)^{\mathrm{c}}$

Clinical status of samples $(n=111)$

Pre-treatment, CR/PR

$59(53 \%)$

Pre-treatment, SD/PD

$16(14 \%)$

$16(14 \%)$

$20(18 \%)$

Post-treatment, non-P
Post-treatment, PD

$84(76 \%)$

$27(24 \%)$

Metastasis

$51.14 \pm 12.71^{\mathrm{d}}$

FPKM

${ }^{a}$ Data presented as N (\%) unless otherwise stated. ${ }^{b}$ Data presented as median (range). ${ }^{\mathrm{c}}$ Data presented as $\mathrm{n}(95 \% \mathrm{CI}) .{ }^{\mathrm{d}}$ Data presented as the mean \pm standard deviation. FOLFIRI, 5-fluorouracil, folinic acid and irinotecan; FOLFOX, 5-fluorouracil, folinic acid and oxaliplatin; MSI-H, microsatellite instability-high; MSI-L, microsatellite instability-low; MSS, microsatellite stable; pMMR, proficient mismatch repair; CI, confidence interval; CR, complete response; PR, partial response; SD, stable disease; PD, progressive disease; FPKM, Fragments Per Kilobase of transcript per million. 
library size $200-400 \mathrm{bp}$ ) were proceeded to sequencing. A total of 113 samples were analyzed, where two samples (one pre-Tx CR/PR and one post-Tx PD) failed to meet the quality assurance criteria (Table SI). Paired-end sequencing with $100 \mathrm{bp}$ per read was conducted using an HiSeq 2500 platform (cat. no. SY-401-2501; Illumina, Inc.). HiSeq PE Rapid Cluster kit v2 (cat. no. PE-402-4002; Illumina, Inc.) and HiSeq Rapid SBS kit v2 (cat. no. FC-402-4021; Illumina, Inc.) were used for the sequencing, with each well loaded with $5 \mathrm{nM}$ of DNA. After sequencing was completed, the raw data were processed using an RNA-seq analysis pipeline. All FASTQ format reads were assessed for quality control using the FASTQC software (v0.11.8) (22). The Illumina sequencing platform-specific adaptors and poor quality read bases were trimmed using Trim Galore (v0.4.5) (23). The trimmed reads were mapped onto the reference genome (human reference genome build version GRCh38/hg38) using STAR aligner (v2.6.0) (24), such that output SAM/BAM files were obtained. The mean of the total reads was 123,416,623 and the GC content per sequence was $47.69 \%$. Each sample had an average of 35,361,303 reads. Gene expression was quantified using RSEM (v1.2.23) (25) and normalized through the DESeq2 BiocManager package (v1.20.0) (26), which derived fragments per kilobase of transcript per Million (FPKM) values for between-sample comparisons.

Differential gene expression analysis (DGE) was performed by using the same DESeq2 package. In DGE analysis, $\log _{2}$ fold change of the normalized abundance between groups was calculated using the DESeq2 package. $\log _{2}$ transformation is commonly used to analyze expressions based on the proportional changes rather than additive changes and to obtain normality of the expression distribution. Using the differential expression (DE) results, which ranked the list of genes and $\log _{2}$ fold-change, gene set enrichment analysis (GSEA) was conducted using the clusterProfiler package (27). In the result of the GSEA, the pathway ID, enrichment score, P-values are listed. The P-values are calculated by testing how frequently the enrichment score in the actual ranking is bigger compared with that in the random permutation. A significant P-value implies that the expressions in the pathway are significantly enriched. Only pathways related to the $N F 1$ (has:4763) and EGFR genes (hsa:1956) were focused upon in the present study. The KEGG pathways related to these genes were retrieved using the following approach: WebDBGET (https://www. genome.jp/dbget-bin/www_bget?-h), an integrated database retrieval system, describes how to retrieve related pathways to a gene by using a specific web URL format along with the gene accession identifier (28). Protocols on this website were followed to search for NF1-related pathways (https://www. genome.jp/dbget-bin/get_linkdb?-t+pathway+hsa:4763) and EGFR-related pathways (https://www.genome.jp/dbget-bin/get_ linkdb?-t+pathway+hsa:1956). In each retrieval site, pathways contained in the 'Pathway' section were used. In the GSEA results, pathways that did not overlap with these KEGG pathways were excluded, where three NF1-related pathways (hsa01521, hsa04014 and hsa04010) and two EGFR-related pathways (hsa05235, hsa01521) were included.

Immunohistochemistry. IHC for NF1 was performed using the IHC-plus ${ }^{\mathrm{TM}}$ polyclonal rabbit anti-human NF1 antibody (1:500; cat. no. LS-B14758; LifeSpan BioSciences, Inc.). All staining procedures were performed using a BenchMark XT automatic immunostaining device (Ventana Medical Systems) according to the manufacturer's protocol. Briefly, antigen retrieval was done by boiling sections using Cell Conditioning 1 buffer (cat. no. 950-124; Ventana Medical Systems, Inc.) for $32 \mathrm{~min}$ at $95^{\circ} \mathrm{C}$. The sections were incubated with the anti-NF1 antibody for $16 \mathrm{~min}$ at $37^{\circ} \mathrm{C}$ in the automatic immunostainer. Signals were visualized using the Ventana OptiView DAB IHC Detection kit (cat. no. 06396500001; Ventana Medical Systems, Inc.): OptiView HQ Linker for $8 \mathrm{~min}$ at $37^{\circ} \mathrm{C}$, Optiview HRP Multimer for $8 \mathrm{~min}$ at $37^{\circ} \mathrm{C}$, OptiView $\mathrm{H} 2 \mathrm{O} 2 / \mathrm{DAB}$ for $8 \mathrm{~min}$ at $37^{\circ} \mathrm{C}$ and OptiView Copper for $4 \mathrm{~min}$ at $37^{\circ} \mathrm{C}$. All specimens were reviewed and scored semi-quantitatively by a pathologist (JK) who gave a score ranging from 0 to 3 . Since the NF1 staining was mostly diffuse, the four-tier scoring system was based on the average staining intensity (1, weak; 2 , moderate; 3 , strong). In addition, 0 was given only when NF1 expression was negligible in all tumor cells. NF1 was regarded to be lost if cytoplasmic staining was absent in tumor cells in the presence of intact expression in the internal non-neoplastic control cells.

NGS test. Targeted sequencing with Oncopanel AMC version 3 was conducted as described in a previous study (29). Only 88 of the 113 samples could be analzyed with RNA sequencing due to insufficient tumor tissues. Briefly, genomic DNA was extracted from formalin-fixed, paraffin-embedded (FFPE) tissue specimens using a NEXprep ${ }^{\mathrm{TM}}$ FFPE Tissue kit (cat. no. NexK-F02T5/NexK-F02TH; Genes Laboratories, Inc.). The quantity and quality of the extracted DNA were examined using a Qubit dsDNA HS Assay kit (Thermo Fisher Scientific, Inc.). Targeted NGS analysis was done using the MiSeqDx or Nextseq 500Dx platforms (Illumina, Inc.) depending on the required sample throughput with Oncopanel AMC version 3, which was designed by Asan Medical Center through SureDesign (https://earray.chem.agilent.com/suredesign/index.htm; Oncopanel AMC version 3 RNA bait; Agilent Technologies,Inc.). This paneltargets 382 genes, including entire exons of 199 genes, 184 hot spots and partial introns for eight genes that were frequently reported to be rearranged in cancer. In total, $200 \mathrm{ng}$ gDNA was used in library preparation with SureSelectXT Reagent kit, HSQ, 96 (cat. no. G9611B, Agilent Technologies, Inc.) and concentration of the target-enriched libraries was measured by quantitative PCR (KAPA SYBR fast qPCR kits; cat. no. 07959362001; Kapa Biosystems; Roche Diagnostics) before loading onto the sequencing platform for paired-end sequencing (sequencing read length $2 \times 75 \mathrm{bp}$; using MiSeq Reagent kit v3; cat. no. MS-102-3001; Illumina, Inc.). Sequenced reads were aligned to the human reference genome (Build 37; National Center for Biotechnology Information) with Burrows-Wheeler Aligner (version 0.5.9; https://sourceforge. net/projects/bio-bwa/postdownload) using default options. PCR duplicates were removed using the MarkDuplicates tool (version 2.20.5; https://broadinstitute.github.io/picard). Base qualities were recalibrated using the GATK BaseRecalibrator tool (version 4.1.5.0; https://software, broadinstitute. org/gatk/download). Somatic single nucleotide variants (SNVs) and short indels were detected with an unmatched normal using 'Mutect' version 1.1.6 and the 'SomaticIndelocator' tool within GATK. Common and germline variants from somatic variant 
candidates were filtered out using the common 'dbSNP' build 141, Exome Aggregation Consortium release 0.3.1 (https://exac. broadinstitute.org) and Korean Reference Genome Database (https://152.99.75.168/KRGDB) and an in-house panel of normal variants. Final somatic variants were annotated using Variant Effect Predictor version 79 (https://m.ensembl. org/info/docs/tools/vep/script/vep_download.html)andconverted to maf file format using vef2maf (GitHub; https://github. $\mathrm{com} / \mathrm{mskcc} / \mathrm{vcf} 2 \mathrm{maf})$.

Statistical analysis. Continuous variables were presented as mean \pm standard deviation from three to eight samples per condition. Student's t-test was performed for two experimental groups evaluated using two-sample equal-variance unpaired t-test. One-way analysis of variance (ANOVA) was performed for multiple experimental groups involving one factor, whereas two-way ANOVA was performed for experiments involving two or three factors. Tukey's multiple comparison test was used as a post-hoc test for ANOVA. $\mathrm{P}<0.05$ was considered to indicate a statistically significant difference. For western blotting analysis, three repeats were performed. For all statistical analysis, GraphPad Prism 7.0 (GraphPad Software, Inc.) and Stata15.1 (StataCorp LP) software were used.

\section{Results}

Basal levels of NF1 expression of CRC cell lines and cetuximab sensitivity. The experimental design of the present study is summarized in Fig. S1. The expression levels of NF1 in the CRC cell lines were first tested. Western blotting (Fig. 1A and B) revealed that the levels of NF1 protein expression is significantly higher in NCI-H508 (KRAS/BRAF $F^{\mathrm{V} 600}$ wild-type) and Caco-2 (KRAS/BRAF ${ }^{\mathrm{V} 600}$ wild-type) cells compared with those in KM12C $\left(K R A S / B R A F^{\mathrm{V} 600}\right.$ wild-type; microsatellite-high; NF1 mutant) and SW480 (KRAS G12V mutant). RT-qPCR also demonstrated the significantly higher expression levels of NF1 mRNA in NCI-H508 and Caco-2 cells compared with those in KM12C and SW480 cells (Fig. 1C). Colony formation assay results demonstrated different numbers of colonies form at baseline despite the same number of cells being seeded under the same condition (Fig. 1D). This may be due to different growth patterns and growth speeds among the four cell lines. However, the relative colony numbers formed by cetuximab-treated cells were significantly reduced compared with those in the control at $100 \mu \mathrm{g} / \mathrm{ml}$ (NCI-H508) or $50 \mu \mathrm{g} / \mathrm{ml}$ (Caco-2), but no significance could be observed in cell lines with little to low NF1 expression (NF1-Low) (Fig. 1E). Cell viability testing also revealed significant growth inhibition by cetuximab in NF1-high cell lines, whilst NF1-low cell lines were found to be intrinsically more resistant to cetuximab (Fig. 1F).

NF1 knockdown with siRNA. To investigate whether the downregulation of NF1 is sufficient to induce resistance to cetuximab, the NF1-high cell lines were transfected with NF1-siRNAs. Both NF1-siRNAs significantly reduced NF1 protein expression in both NCI-H508 and Caco-2 cells (Fig. 1A-C). Elevations in the phosphorylation levels of MEK and ERK was prominent in NF1-knockdown cells compared with cells transfected with scrambled-siRNA, but total MEK and ERK expression were not changed in both NCI-H508 and Caco- 2 cells (Fig. 2A-C). The significantly reduced expression of NF1 mRNA after siRNA transfection compared with those in cells transfected with scrambled siRNA was also confirmed by RT-qPCR (Fig. 2D). The cell lines transfected with NF1 siRNA demonstrated a change into a more attached growth pattern and a significantly greater number of cells compared with those in cells transfected with scrambled siRNA (Fig. 2E). Data from the DAPI assay indicated lower levels of cetuximab-induced apoptosis by observing fewer apoptotic bodies, referred to as extracellular vesicles containing fragmented nucleus component with intense staining by DAPI, in cells transfected with NF1-siRNA than those in cells transfected with scrambled siRNA (Fig. 2F). In the presence of cetuximab, western blotting of apoptosis markers in both cell lines also showed significantly decreased cleavage of caspase 3 and PARP after transfection with NF1-siRNA compared with that in cells transfected with scrambled siRNA (Fig. 2G-I).

NF1-GRD plasmid expression. Subsequently, NF1-Low cell lines were tested for the physiological effects of NF1 overexpression. KM12C and SW480 cells were transfected with the NF1-GRD plasmid (Fig. 3A). NF1-GRD mRNA expression was significantly increased in NF1-GRD-transfected cells compared with that in cells transfected with empty vector in both KM12C and SW480 cells (Fig. 3B). The expression of total NF1 protein also increased in NF1-GRD-transfected cells compared with that in empty vector-transfected cells in all NF1-Low cells (Fig. 3C). The phosphorylation of MEK and ERK was significantly decreased by NF1-GRD overexpression in both cell lines compared with that in cells transfected with the empty vector (Fig. 3C-E). NF1-GRD overexpression also inhibited cell proliferation in KM12C and SW480, as shown by a significantly lower number of cells in the NF1-GRD overexpression group compared with those cells transfected with the empty vector (Fig. 3F) Furthermore, NF1-GRD overexpression was sufficient to potentiate cetuximab-induced apoptosis in these resistant cell lines, which was supported by the increased cleavage of caspase and PARP according to western blotting (Fig. 3G-I). DAPI assay also showed increased nuclear fragmentation in cells overexpressing NF1-GRD transfection compared with that in cells transfected with the empty vector but not treated with cetuximab (Fig. 3J). However, this phenomenon was more prominent following cetuximab treatment (Fig. 3J).

NF1 mRNA expression in tumor samples. After investigating the in vitro relationship between NF1 expression and cetuximab sensitivity, the levels of $N F 1$ transcript expression in clinical CRC samples was then estimated. To explore how different quantities of $N F 1$ transcripts are associated with the clinical outcomes of patients treated with anti-EGFR, $111 R A S$ and $B R A F^{\mathrm{V} 600}$ wild-type CRC samples from 92 patients (19 patients provided two samples and 73 patients provided one sample) who received cetuximab as palliative chemotherapy were analyzed using RNA sequencing. Patient characteristics are listed in Table I. In the present study, the majority of patients underwent first-line treatment with cetuximab and combination chemotherapy (82\%), were diagnosed with left-sided CRC 

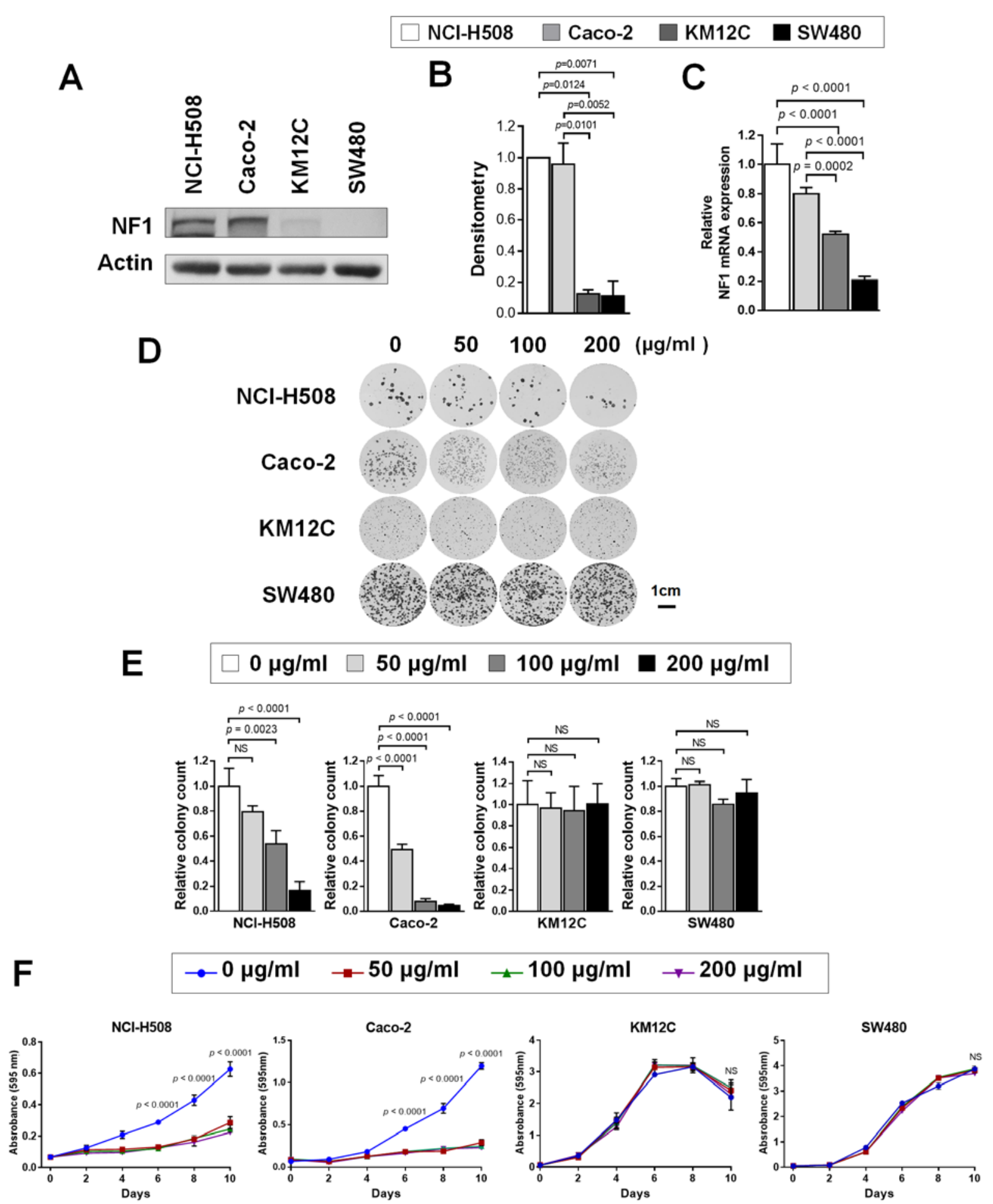

Figure 1. CRC cell lines with resistance to cetuximab shows lower NF1 expression levels. (A) Western blotting was used to measure NF1 expression in NCI-H508, Caco-2, KM12C and SW480 cells. (B) Relative expression of NF1 by densitometric quantification of the western blotting images in (A). P<0.05; $\mathrm{n}=3$. (C) NF1 mRNA expression in NCI-H508, Caco-2, KM12C and SW480 cells. P<0.001, n=3. (D) NCI-H508, Caco-2, KM12C and SW480 cells were cultured with $0,50,100$, and $200 \mu \mathrm{g} / \mathrm{ml}$ cetuximab for 10 days and stained with crystal violet before imaging. (E) The number of surviving colonies at each cetuximab concentration were normalized to the control and plotted. $\mathrm{P}<0.01, \mathrm{n}=3$. (F) The cells were cultured with $0,50,100 \mathrm{and} 200 \mu \mathrm{g} / \mathrm{ml}$ of cetuximab for $0,2,4,6,8$ and 10 days, stained with crystal violet and assayed for cell growth. $\mathrm{P}<0.001$. Actin was used as the loading control as the housekeeping gene for use in western blot analysis. GAPDH was used as the housekeeping gene for reverse transcription-quantitative PCR. NF1, neurofibromin; siRNA, small-interfering RNA; CRC, colorectal cancer.

(79\%) and stage IV disease (89\%). In total, 75 pre-treatment samples (69 from primary tumors and six from metastatic organs) and 36 post-treatment samples (15 from primary tumors and 21 from metastatic organs) were analyzed. The majority of samples (104 samples) were obtained at the time of metastatic spread except for seven pre-treatment samples, which were obtained from stage I-III surgical specimens before recurrence. The FPKM values of NFI were then 

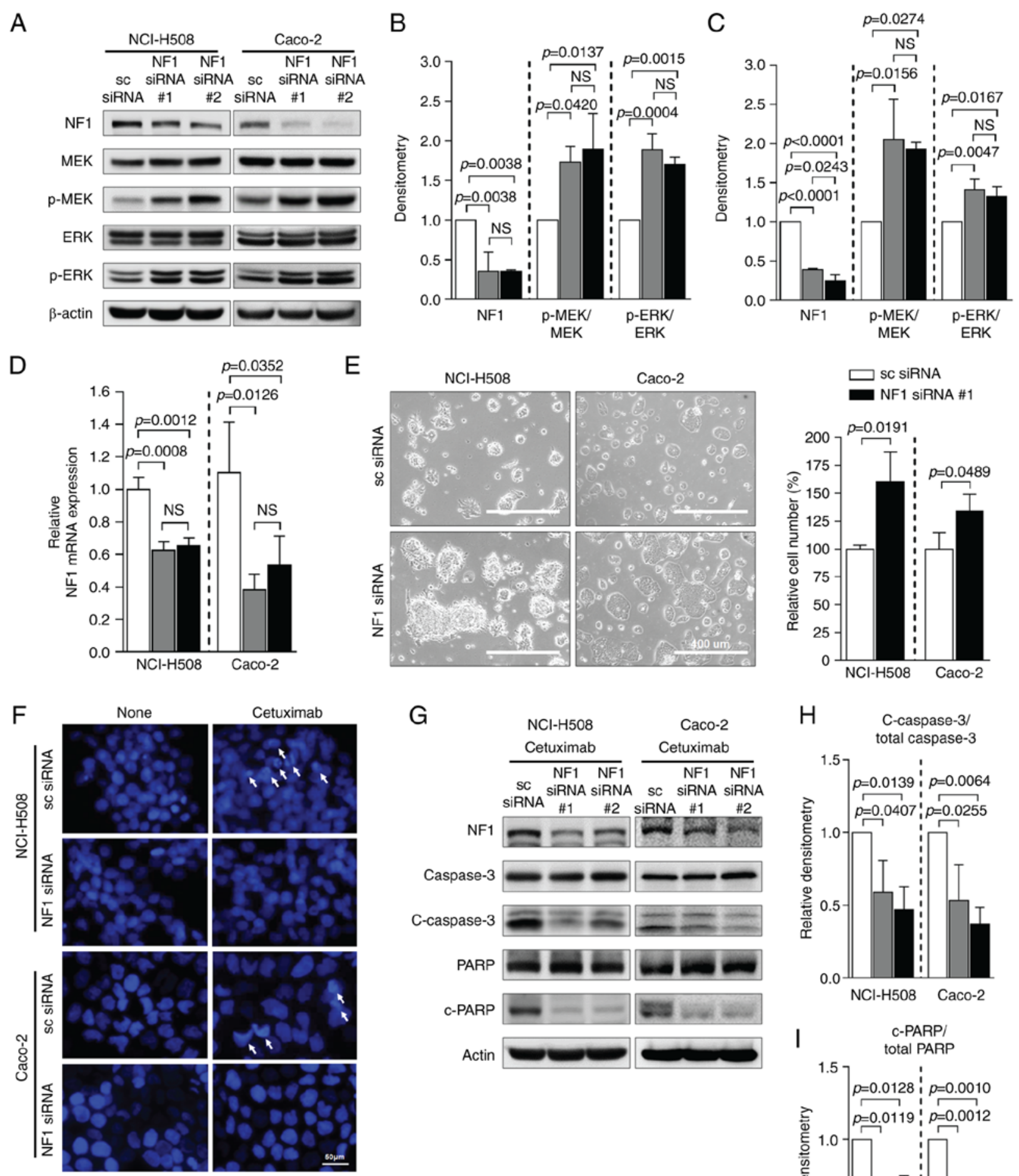

$\mathrm{H} \quad \begin{gathered}\text { C-caspase-3/ } \\ \text { total caspase-3 }\end{gathered}$
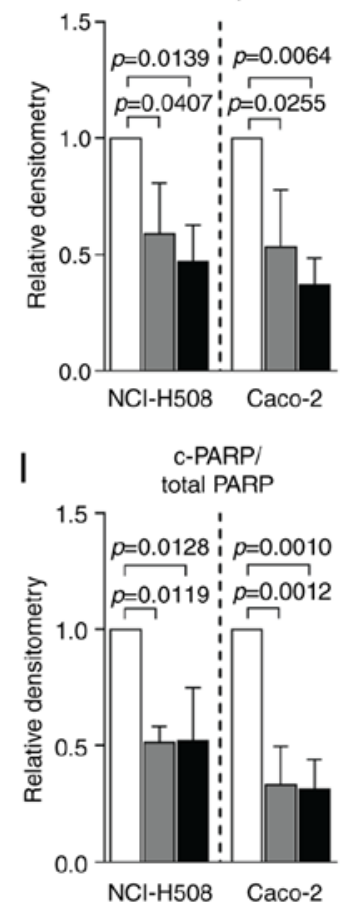

Figure 2.NF1 suppression causes resistance to cetuximab in CRC cell lines expressing NF1. Cells were transfected with either scrambled-siRNA or NF1-siRNA. (A) Western blot analysis of the expression of NF1 and the activation levels of MEK and ERK. Relative expression of NF1 and the ratio of p-MEK/total MEK and p-ERK/total ERK in (B) NCI-H508 and (C) Caco-2 cells were quantified using densitometry of images in (A). (D) mRNA expression level of NF1 in two CRC cell lines transfected with NF1-siRNA. (E) EVOS microscope images of NCI-H508 and Caco-2 cells transfected with scrambled siRNA or NF1 siRNA with the relative number of cells measured using the ImageJ software. Magnification, x200. Cells transfected with NF1 siRNA showed more proliferative and aggregative features. (F) DAPI staining assay of NCI-H508 and Caco-2 cells after NF1 siRNA transfection and cetuximab treatment. (G) Protein expression of apoptosis markers c-caspase 3 and c-PARP. Relative densities of (H) c-caspase 3/total caspase 3 and (I) c-PARP/total PARP were quantified. Actin was used as a loading control for use in western blot analysis. GAPDH was used as the housekeeping gene for reverse transcription-quantitative PCR. P $<0.05$, one-way ANOVA. NF1, neurofibromin 1; sc, scrambled; p-, phosphorylated; c-, cleaved; PARP, poly-(ADP ribose) polymerase; si, small interfering. 
A

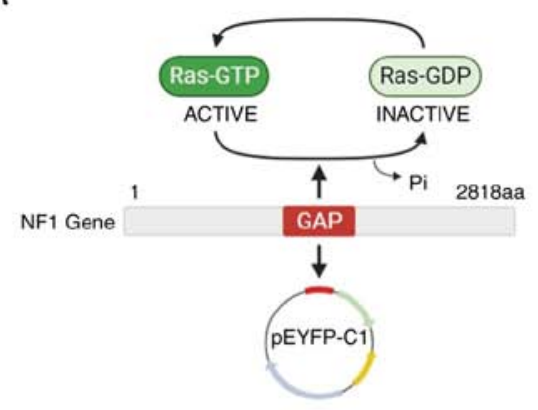

$\square$ Empty $\quad$ NF1-GRD

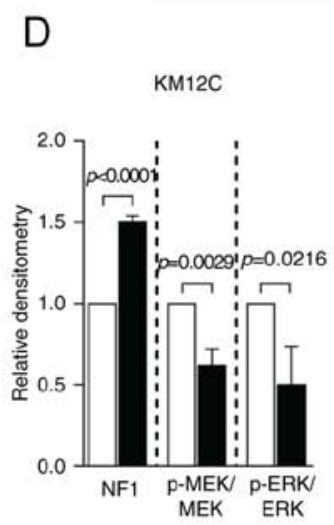

E

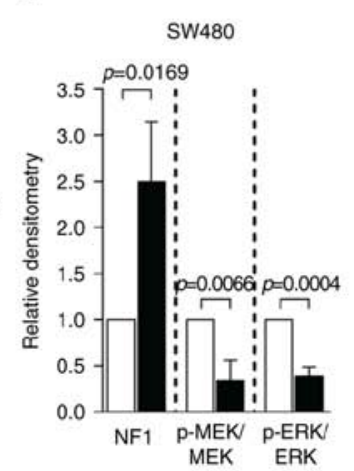

B

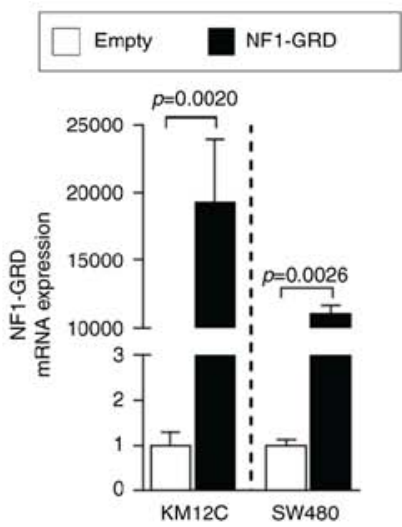

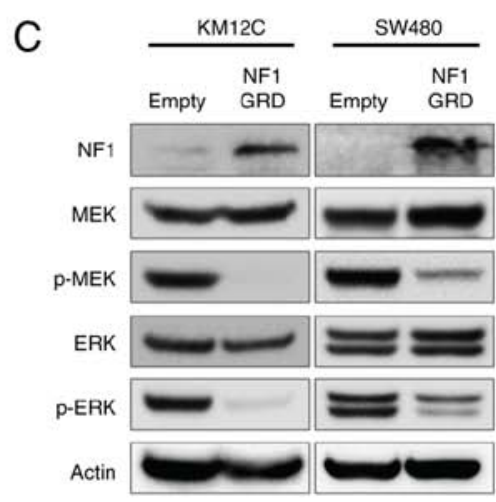

F
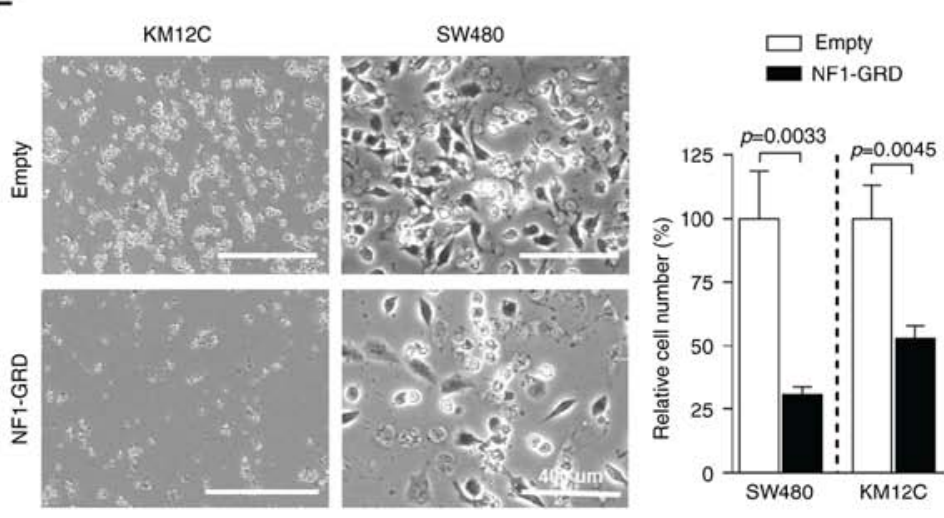

$\mathrm{H}$

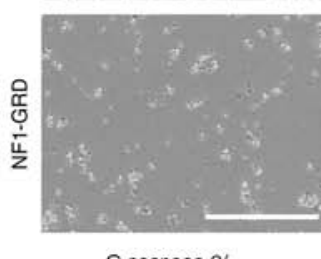

C-caspase-3/

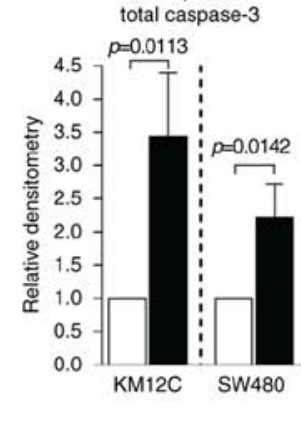

J

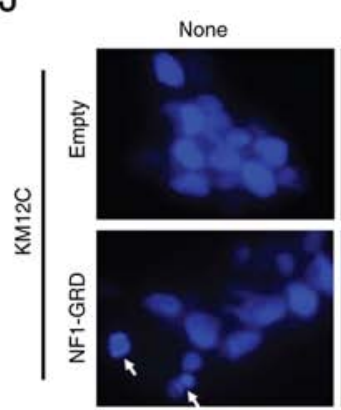

Cetuximab

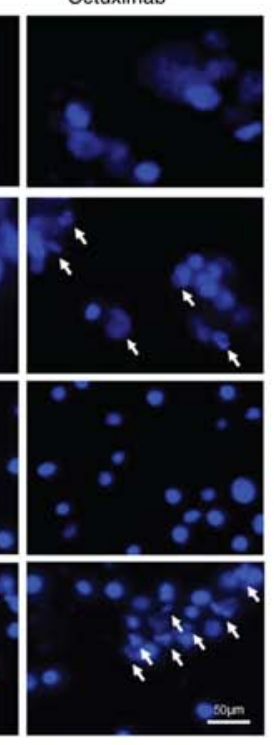

I

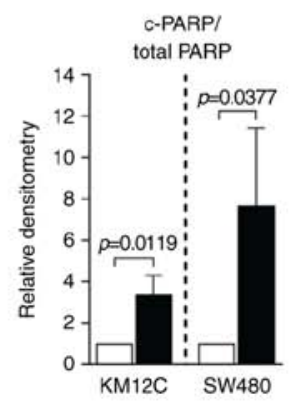

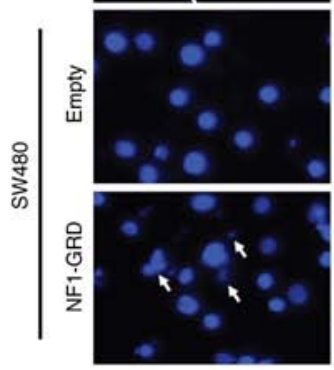

G

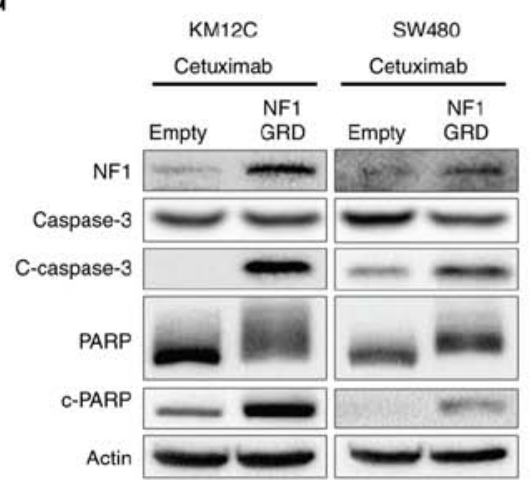


A

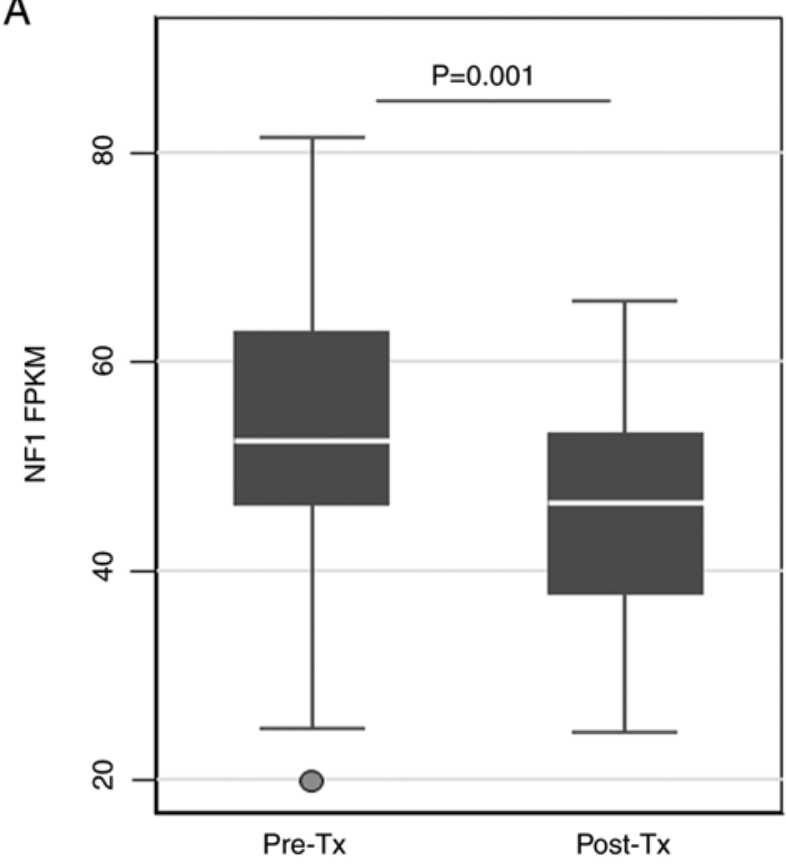

B

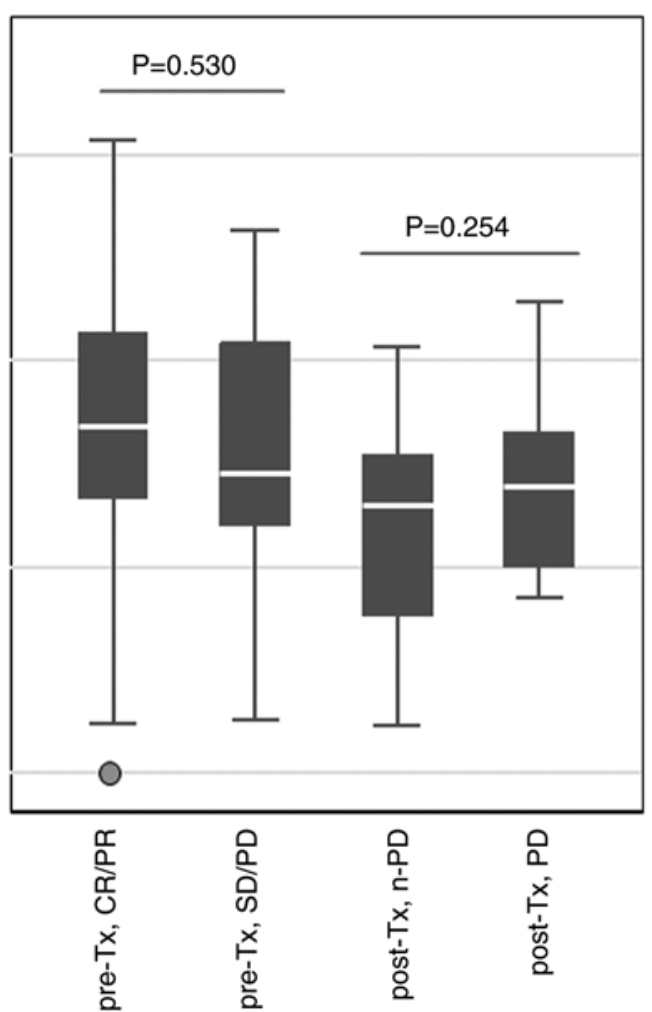

Figure 4. NF1 FPKM values in cetuximab-treated tumor samples. NF1 FPKM values were displayed according to the cetuximab treatment status. (A) FPKM values after sorting by timepoint at which tumor samples were obtained. (B) FPKM values after sorting by combining the treatment response and time points at which tumor samples were obtained. NF1, neurofibromin; FPKM, fragments per kilobase of transcript per million; Tx, treatment; CR, complete response; PR, partial response; $\mathrm{SD}$, stable disease; $\mathrm{PD}$, progressive disease.

differences between pre-Tx CR/PR and SD/PD or between post-Tx non-PD and PD (Fig. 4B). There were also no significant associations between the NF1 FPKM values and other clinical characteristics, namely treatment lines, side of tumor sample origin or whether the sample was from the primary tumor or a metastasis (Fig. S2).

Among the 111 samples, $88(79 \%)$ were also analyzed with NGS, which revealed only two (2.2\%) samples showing non-synonymous NF1 mutations. One post-treatment sample obtained at PD showed a truncating mutation (K918Gfs*17) with the variant allele frequency of 0.11 , of which the NF1 FPKM value was 52.6, whilst its paired pre-treatment sample did not harbor the NF mutation (the NF1 FPKM value was 56.7). The other was a missense mutation (p.T1627A) with the allele frequency of 0.46 , and its NF1 FPKM value was 74.9. It was found in a pre-treatment sample that showed clinical CR, not matched with a post-treatment sample.

Subsequently, the expression profiles of NF1 and EGFR signaling components were analyzed according to the clinical status of the tumor samples. For differentially expressed gene analysis using the RNA sequencing data, pathways containing 'NF1 (entrez ID: 4763)' or 'EGFR (entrez ID: 1956)' were selected in the pathway names or pathway gene set. Pathways in the pathway sets defined as NF1-related pathways or EGFR-related pathways by the KEGG pathway database were also selected. After this selection procedure, three NF1-related pathways (hsa01521, hsa04014 and hsa04010) and two EGFR-related pathways (hsa05235 and hsa01521) were found in the GSEA results (Table SII). In all of these pathways found, the GSEA P-values were not significant, meaning that expressions in these pathways were not significantly enriched.

Frequency of NFI mutation in CRC and NFI protein expression associated with mutation. In a total of the 1,449 patients with CRC who underwent the clinical NGS test, 29 truncated mutations in the NF1 gene (nonsense, frameshift, or splice site) were found in $26(1.8 \%)$ patients (Table SIII). The majority of the allele frequencies were $<0.5$, whilst only five cases showed allele frequencies $>0.5$, where four cases were found with frameshift mutations and one with splice-site mutations $(5 / 1,449,0.3 \%)$. In addition, one of the tumors harboring those high allele frequencies of $N F 1$ frameshift mutations (CRC-1213) showed the absent expression of NF1 in tumor cells but adjacent normal colonic crypts were positive for NF1 (Fig. 5A), whilst tumors with lower NF1 mutation allele frequencies showed comparable NF1 expression levels compared with tumors with wild-type NF1 (Fig. 5B and C).

\section{Discussion}

The present study showed that reduced NF1 expression may be associated with anti-EGFR antibody resistance in CRC, where the level of expression was generally downregulated after treatment, implicating a role for NF1 in the acquisition of resistance to anti-EGFR therapy. The NF1 gene is located on the $17 \mathrm{q} 11.2$ locus and is relatively large in terms of genomic size $(350 \mathrm{kbp})$, which also has a complex structure consisting of 61 exons (30). In addition, mutations are 
A

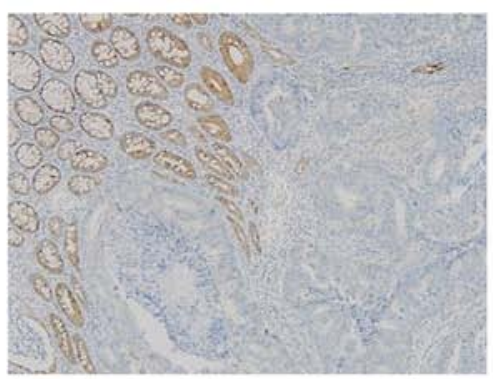

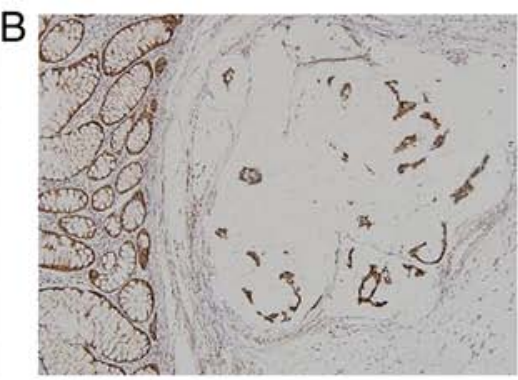

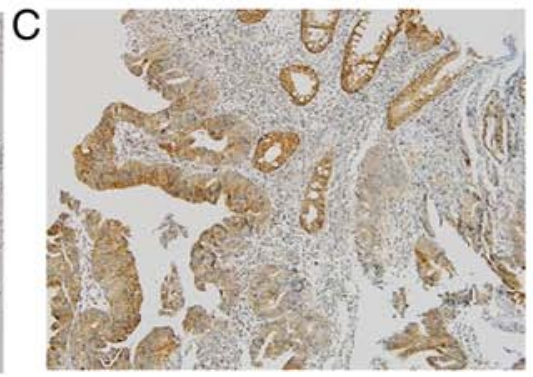

Figure 5. Representative images of NF1 expression by immunohistochemical staining in tissues from patients with colorectal cancer. (A) Tumor with high VAF NF1 mutation (CRC-1213). (B) Tumor with low VAF NF1 mutation (CRC-1114). (C) NF1 wild-type tumor (CRC-542). Magnification, x200. NF-1, neurofibromin; VAF, variant allele frequency.

generally spread over all regions without specific clusters of recurrent mutations (30). Pathogenic germline mutations of NFI are known to cause the neurofibromatosis type 1 disease, which is an inherited syndrome that increases the predisposition of developing tumors, including neurofibromas, malignant peripheral nerve sheath tumors, optic gliomas, rhabdomyosarcomas and neuroblastomas (13). Furthermore, somatic mutations or other molecular aberrations in the $N F 1$ gene in non-NF1-associated sporadic tumors, such as melanoma (14), lung cancer $(31,32)$, breast cancer $(33,34)$, or CRC $(9,35)$, have been previously investigated, where their roles in resistance to targeted agents or hormonal therapy are being elucidated (30). In CRC, RAS/MAPK dysregulation occurs in $\sim 80 \%$ microsatellite-stable subtypes and $60 \%$ microsatellite-unstable subtypes and is a crucial factor of anti-EGFR resistance (36). Therefore, $N F 1$ aberrations may hold have potential predictive values.

Notable differences were observed in the NF1 protein expression levels in the CRC cell lines according to their genotype and sensitivity to cetuximab. Cetuximab-sensitive cell lines NCI-H508 and Caco-2 were of the $R A S / B R A F^{\mathrm{v} 600}$ wild-type genotype and microsatellite stable without $N F 1$ mutations, which showed higher NF1 expression. By contrast, cetuximab-resistant cell lines with lower NF1 expression levels were found to harbor KRAS mutations (SW480) or the microsatellite-unstable genotype with $N F 1$ frameshift mutation (KM12C) (17). Downregulation of NF1 expression in $\mathrm{KM} 12 \mathrm{C}$ may be explained by the presence of the $N F 1$ truncating mutation (T676fs; allele frequency, 0.44). In SW480 cells, reduced NF1 expression may be associated with the KRAS G12V mutant protein, which is constitutively active irrespective of its upstream signal, EGFR (37). Codon 12 $K R A S$ mutant protein is known to inhibit NF1 by forming nonproductive binding so that wild-type KRAS is activated during the scarcity of NF1 (38).

In cetuximab-sensitive cell lines with high NF1 expression, suppression of NF1 expression was sufficient for inducing resistance to cetuximab, which also resulted in enhanced MEK and ERK signaling downstream and reduced apoptosis. These findings are in line with those from previous studies, which demonstrated that siRNA- and genome-side CRISPR-mediated NFI inactivation induced cetuximab resistance in $R A S$ and $B R A F$ wild-type CRC cell lines $(9,38,39)$. Conversely, it was also demonstrated that NF1-GRD overexpression in NF1-low cell lines reversed their intrinsic resistance to cetuximab whilst also attenuating ERK and MEK signaling. In addition, the expression levels of the apoptosis markers were elevated in the cetuximab-resistant cell lines transfected with the NF1-GRD plasmid. This phenomenon could also be observed in the KRAS mutant cell line SW480, suggesting that overcoming the intrinsic resistance of $R A S$-mutant CRC to cetuximab can be achieved by restoring NF1 expression. These results are consistent with those reported by a recent study, which revealed the role of NF1 interaction with mutant KRAS protein in anti-EGFR resistance (38). The study showed the levels of wild-type RAS-GTP expression, rather than mutant RAS-GTP, was related to resistance to cetuximab in various types of KRAS-mutant CRC cell lines, and the cell lines harboring the KRAS codon 12 mutation showed higher levels of wild-type RAS-GTP compared with those with $K R A S$ wild-type or G13D KRAS mutants, However, NF1 transfection rendered the $\mathrm{G} 12 \mathrm{~V}$ mutant cell line sensitive to cetuximab due to the hydrolysis of abundant wild-type RAS-GTP by NF1. This suggests that sensitivity to anti-EGFR treatment in KRAS mutant tumors can be enhanced by NF1 overexpression (38).

The NF1 mRNA expression levels in $R A S$ and $B R A F^{\mathrm{V} 600}$ wild-type CRC tumor samples obtained before or after cetuximab treatment were next analyzed to test the association between NF1 expression and the response to cetuximab. Pre-treatment samples showed relatively higher NF1 transcripts compared with those in their post-treatment counterparts, whilst response to cetuximab was not significantly associated the pre-treatment baseline $N F 1$ transcript levels. This suggests that the heterogeneity in the NFI expression levels within the $R A S$ and $B R A F^{\mathrm{V} 600}$ wild-type CRC group may not accurately predict the response to cetuximab. However, exposure to cetuximab may be associated with lower expression levels of NF1. This is in line with the findings from a previous study in non-small-cell lung cancer tumor samples, which identified NF1 as a determinant of EGFR tyrosine kinase inhibitor resistance using genome-wide siRNA screening (8). This previous study also found reduced NF1 mRNA expression in post-treatment samples with acquired resistance (8). However, GSEA analysis in the present study did not reveal any differences in the expression of EGFR, RAS or MAPK signaling pathway components between pre- and post-treatment samples or between post-treatment non-PD and PD samples. Therefore, the slightly reduced NFI expression in the post-treatment samples may not be associated with a significant change in 
the transduction of growth signals through the EGFR/MAPK pathway.

The mechanism underlying the reduced expression of NF1 transcripts in the post-treatment samples remains unclear. In breast cancer, $N F 1$ truncating mutations, such as frameshift, nonsense or stop-gain, which were absent in primary tumors, became emergent in metastatic tumor tissues in $\sim 3 \%$ patients and was associated with resistance to endocrine therapy (34). However, previous studies that conducted NGS analysis on cetuximab-treated CRC samples revealed no evidence of recurrently acquired $N F 1$ mutations with sufficient allele fraction $(9,40)$. Therefore, another transcriptomic or epigenetic mechanism other than gene regulation may be responsible for the reduced NF1 transcripts in post-treatment samples.

In the present study, mutations in $N F 1$ in the cetuximab-treated samples were rare, where the NF1 FPKM did not seem to associate with NF1 mutations. Only two of the 88 samples that were tested for both RNA sequencing and NGS revealed NF1 mutations. One was a frameshift mutation that emerged after cetuximab treatment. However, its NFI expression was not downregulated since the NF1 FPKM value associated with this mutation was comparable with the mean value of the entire cohort. Its lower allele frequency (0.11) implies that this frameshift mutation is a heterozygous variant that is insufficient to suppress the function of a tumor suppressor gene such as NF1. Another mutation (p.T1627A) found in the pre-treatment sample was annotated as having uncertain significance in ClinVar (https://www.ncbi.nlm.nih. gov/clinvar/variation/231988/). This variant may not have functional implications, considering the high NF1 FPKM value and the favorable clinical response to cetuximab exhibited by the patient.

After the finding that mutations in the NF1 gene may not be related to NF1 expression, the prevalence and type of NF1 mutations in a larger genomic dataset of CRC in Asan Medical Center were investigated. Only $1.8 \%$ patients with CRC had truncating mutations in NF1, which corresponded to the prevalence reported by the American Association For Cancer Research Project Genomics Evidence Neoplasia Information Exchange (2.3\%; 144/6,303) (41). Previous studies also reported the rate of somatic mutations in the $N F 1$ gene to be ranging from 3.8 to $6.25 \%$ in CRC $(30,36,42)$. However, this includes all types of mutations, of which a large proportion may have been missense mutations of unknown significance. Although any types of mutation could in theory result in a pathogenic impact on the function of the NF1 gene, the high likelihood of oncogenicity lies in the truncating mutations of this tumor-suppressor gene (43). This was the reason for truncating mutations in NFI in CRC being focused upon in the present study. The majority of the mutations were of low allele frequencies, which implicates heterozygous mutations. Although mono-allelic loss of NFI has the potential for de novo tumorigenesis, these effects are reportedly limited to benign tumors (44) and known to be insufficient for malignant transformation (45). IHC analysis in the present study revealed that the levels of NF1 protein expression in tumors harboring the heterozygous NF1 mutation were comparable with those in the NFl wild-type. In addition, only $0.3 \%$ of all patients with CRC had a sufficient allele frequency of the NFl truncating mutation that may have an impact on protein expression and possibly drug resistance (45). Taken together, loss of NF1 proteins by inactivating mutations may be a rare phenomenon in CRC.

Several studies have previously demonstrated the association between the efficacy of cetuximab and NF1 mutation (9,35). Mei et al (35) reported that patients with CRC having any single nucleotide variants or insertion/deletion mutations of $N F 1$ or SMAD4 showed poorer progression-free survival after cetuximab treatment compared to those with wild-type NF1 and SMAD4 (35). However, the NF1 variants detected in the present study population also had low allele frequencies $(1.9-7.5 \%$ ), where only one of the three were detected to have NFI mutations, which were frameshift mutations annotated as pathogenic in ClinVar (https://www. ncbi.nlm.nih.gov/clinvar/). In addition, another study showed that intrinsic resistance to cetuximab in CRC was associated with the NF1 truncating mutation combined with the loss of heterozygosity in a small subset of patients (9), suggesting that a 'two-hit hypothesis' can be applied to explain anti-EGFR resistance associated with $N F 1$ mutation. In the present study, none of the five patients who had sufficient allele frequencies of truncating mutations were treated with cetuximab, meaning that analysis of their association with anti-EGFR resistance was not possible due to their rarity. Taken together, mutations causing the biallelic inactivation of NFl do occur in CRC but are relatively rare. Therefore, larger groups of samples containing NGS results are required to test the statistical significance in the association between the mutations and response to anti-EGFR treatment.

A tumor suppressor gene is typically not regarded to be druggable. Therefore, restoration of the functions of these genes is difficult. However, various strategies, including gene therapy and small molecule inhibitors, have been attempted (46). Although gene therapy for tumor suppressor genes is in the early stages of development and the size of NF1 is large for loading into gene delivery vectors, a recent study has shown that the NF1-GRD subunit delivered by adeno-associated virus vectors was sufficient to inhibit RAS activity in malignant peripheral nerve sheath tumor cell lines (47). In addition, nanoparticle platform-mediated delivery of p53 mRNA was shown to induce tumor regression in animal models of hepatocellular carcinoma and non-small-cell lung cancer $(48,49)$. These novel strategies may yet provide alternative therapeutic strategies for anti-EGFR-resistant tumors by restoring NF1 expression in the future.

The present study has several limitations. The effects of NF1 knockdown and overexpression on the apoptosis of all cell lines used in this study were not assessed, This was because the degree of knockdown in NF1-Low cell lines and the degree of overexpression in NF1-High cell lines were not significant (data not shown). However, these experiments could have revealed the relative impact of NF1 expression modulation compared with other cell lines that have different levels of NF1 expression and cetuximab sensitivity. For example, by comparing the effects of NF1 knockdown with NF1-Low and the effects of NF1 overexpression with NF1-High. In addition, apoptosis induced by cetuximab in the present study was demonstrated by measuring the cleavage of caspase 3/PARP through western blotting and imaging of apoptotic bodies using DAPI staining. 
This could have been supported by demonstrating DNA fragmentation using TUNEL assay and by showing early changes in the membrane potential due to apoptosis using Annexin V assay. The tumor samples with NF1 homozygous truncating mutations are so rare that none of the studied patients underwent anti-EGFR Ab treatment. Therefore, the association between NF1 mutation and response to anti-EGFR treatment could not be analyzed. RNA sequencing was performed for RNA extracted from formalin-fixed paraffin-embedded tissues, which is not an ideal source for RNA sequencing due to fragmentation and chemical modification of RNA (50). Therefore, it is generally associated with higher risk of the inadequate quality of RNA. In addition, due to the lack of sufficient tissue quantities, the RNA sequencing results for NF1 expression on those cetuximab-treated samples could not be completely supported with IHC. The clinical characteristics of tumors analyzed using RNA-seq were found to be heterogeneous within the limited number of samples. A number of samples were obtained before the development of metastases, which may not represent the transcriptome of metastatic disease. Therefore, the possibility that other clinical factor, including sidedness or sample origin, could be associated with NF1 expression even though the associations between NFI FPKM and other factors were not statistically significant in the present study (data not shown). The finding of the reduced NF1 mRNA expression in cetuximab-treated tumor samples was not supported by EGFR/RAS/MAPK pathway activation in GSEA, which was not directly proven through any cell lines or animal models in the present study. Therefore, the causal relationship between acquired resistance to cetuximab and NF1 downregulation was not analyzed by this study, which warrants further investigation.

In conclusion, the present study showed that reduced NF1 expression may serve a role in anti-EGFR resistance in CRC cell lines, which could be overcome by restoring NF1 expression. However, its utility as a biomarker is limited in clinical samples. Baseline NF1 expression in $R A S$ and $B R A F^{\mathrm{V} 600 \mathrm{E}}$ wild-type tumors was not related to response to anti-EGFR therapy, but in post-treatment samples NF1 expression tended to be slightly lower than that in their pre-treatment counterparts, suggesting that acquired resistance could be related to NF1 downregulation during treatment. However, the majority of NF1 mutations in $\mathrm{CRC}$ in the present study were heterozygous variants, which did not impact NF1 expression. Baseline NF1 mutation or expression levels do not seem to be relevant biomarkers for predicting anti-EGFR response in CRC. Nevertheless, NF1 expression in post-treatment samples warrants further study to decipher the mechanism underlying anti-EGFR resistance.

\section{Acknowledgements}

The authors would like to thank Professor Seon-Yong Jeong, Department of Medical Genetics, Ajou University School of Medicine (Suwon, South Korea), for providing NF1-GRD constructs for the present study.

\section{Funding}

The present study was supported by the Bio \& Medical Technology Development Program of the National Research Foundation of Korea funded by the Ministry of Science and
ICT (grant no. 2017M3A9B6061825) and was also supported by a grant from the Asan Institute for Life Sciences, Seoul, Republic of Korea (2016-735).

\section{Availability of data and materials}

The data presented in this study will be provided upon request from the corresponding author. RNA sequencing data has been deposited in Gene Expression Omnibus (GSE 183984; https://www.ncbi.nlm.nih.gov/geo/query/acc. cgi?acc=GSE183984).

\section{Authors' contributions}

Conceptualization: EYT, JEK, YSH, SYK and TWK. Data curation: BH (annotated the RNA sequencing data), HDK (annotated the RNA sequencing) and CSJ (scrubbed and maintained the RNA sequencing data). Formal analysis: EYT, MHK, JHK, BH, HDK, CSJ and SYK. Investigation: EYT (performed experiment), MK (performed experiment), YGC (performed experiment), SC (performed experiment) and JHK (performed experiment). All authors have read and agreed to the published version of the manuscript. EYT and SYK confirm the authenticity of all the raw data.

\section{Ethics approval and consent to participate}

A part of the present study (Analysis of patient samples) was approved by the Institutional Review Board of Asan Medical Center (approval no. 2011-0511) and was conducted in accordance with the tenets of the Declaration of Helsinki and Good Clinical Practice. All participants of the present study provided written informed consent for the use of their tumor samples for research purposes.

\section{Patient consent for publication}

Not applicable.

\section{Competing interests}

The authors declare that they have no competing interests.

\section{References}

1. Sung H, Ferlay J, Siegel RL, Laversanne M, Soerjomataram I, Jemal A and Bray F: Global cancer statistics 2020: GLOBOCAN estimates of incidence and mortality worldwide for 36 cancers in 185 countries. CA Cancer J Clin 71: 209-249, 2021.

2. Venook AP, Niedzwiecki D, Lenz HJ, Innocenti F, Fruth B, Meyerhardt JA, Schrag D, Greene C, O'Neil BH, Atkins JN, et al: Effect of first-line chemotherapy combined with cetuximab or bevacizumab on overall survival in patients with KRAS wild-type advanced or metastatic colorectal cancer: A randomized clinical trial. Jama 317: 2392-2401, 2017.

3. Kim SY and Kim TW: Current challenges in the implementation of precision oncology for the management of metastatic colorectal cancer. ESMO Open 5: e000634, 2020.

4. Zhao B, Wang L, Qiu H, Zhang M, Sun L, Peng P, Yu Q and Yuan X: Mechanisms of resistance to anti-EGFR therapy in colorectal cancer. Oncotarget 8: 3980-4000, 2017.

5. Martinelli E, Ciardiello D, Martini G, Troiani T, Cardone C, Vitiello PP, Normanno N, Rachiglio AM, Maiello E, Latiano $\mathrm{T}$, et al: Implementing anti-epidermal growth factor receptor (EGFR) therapy in metastatic colorectal cancer: Challenges and future perspectives. Ann Oncol 31: 30-40, 2020. 
6. Li QH, Wang YZ, Tu J, Liu CW, Yuan YJ, Lin R, He WL, Cai SR, He YL and Ye JN: Anti-EGFR therapy in metastatic colorectal cancer: Mechanisms and potential regimens of drug resistance. Gastroenterol Rep (Oxf) 8: 179-191, 2020.

7. Ratner N and Miller SJ: A RASopathy gene commonly mutated in cancer: The neurofibromatosis type 1 tumour suppressor. Nat Rev Cancer 15: 290-301, 2015.

8. de Bruin EC, Cowell C, Warne PH, Jiang M, Saunders RE, Melnick MA, Gettinger S, Walther Z, Wurtz A, Heynen GJ, et al Reduced NF1 expression confers resistance to EGFR inhibition in lung cancer. Cancer Discov 4: 606-619, 2014.

9. Woolston A, Khan K, Spain G, Barber LJ, Griffiths B, Gonzalez-Exposito R, Hornsteiner L, Punta M, Patil Y, Newey A, et al: Genomic and transcriptomic determinants of therapy resistance and immune landscape evolution during Anti-EGFR treatment in colorectal cancer. Cancer Cell 36 : 35-50.e39, 2019.

10. Cancer Genome Atlas Research Network: Comprehensive genomic characterization of squamous cell lung cancers. Nature 489: 519-525, 2012

11. Cancer Genome Atlas Network: Comprehensive molecular portraits of human breast tumours. Nature 490: 61-70, 2012.

12. Krauthammer $M$, Kong $Y$, Bacchiocchi A, Evans $P$, Pornputtapong N, Wu C, McCusker JP, Ma S, Cheng E, Straub R, et al: Exome sequencing identifies recurrent mutations in NF1 and RASopathy genes in sun-exposed melanomas. Nat Genet 47: 996-1002, 2015.

13. Yap YS, McPherson JR, Ong CK, Rozen SG, Teh BT, Lee AS and Callen DF: The NF1 gene revisited -from bench to bedside. Oncotarget 5: 5873-5892, 2014.

14. Whittaker SR, Theurillat JP, Van Allen E, Wagle N, Hsiao J, Cowley GS, Schadendorf D, Root DE and Garraway LA: A genome-scale RNA interference screen implicates NF1 loss in resistance to RAF inhibition. Cancer Discov 3: 350-362, 2013.

15. Mendes-Pereira AM, Sims D, Dexter T, Fenwick K, Assiotis I, Kozarewa I, Mitsopoulos C, Hakas J, Zvelebil M, Lord CJ and Ashworth A: Genome-wide functional screen identifies a compendium of genes affecting sensitivity to tamoxifen. Proc Natl Acad Sci USA 109: 2730-2735, 2012.

16. Hölzel M, Huang S, Koster J, Ora I, Lakeman A, Caron H, Nijkamp W, Xie J, Callens T, Asgharzadeh S, et al: NF1 is a tumor suppressor in neuroblastoma that determines retinoic acid response and disease outcome. Cell 142: 218-229, 2010.

17. Berg KCG, Eide PW, Eilertsen IA, Johannessen B, Bruun J, Danielsen SA, Bjørnslett M, Meza-Zepeda LA, Eknæs M, Lind GE, et al: Multi-omics of 34 colorectal cancer cell lines-a resource for biomedical studies. Mol Cancer 16: 116, 2017.

18. Park HJ, Lee SJ, Sohn YB, Jin HS, Han JH, Kim YB, Yim H and Jeong SY: NF1 deficiency causes Bcl-xL upregulation in Schwann cells derived from neurofibromatosis type 1-associated malignant peripheral nerve sheath tumors. Int $\mathbf{J}$ Oncol 42 : 657-666, 2013.

19. Schneider CA, Rasband WS and Eliceiri KW: NIH image to imageJ: 25 years of image analysis. Nat Methods 9: 671-675, 2012.

20. Eisenhauer EA, Therasse P, Bogaerts J, Schwartz LH, Sargent D, Ford R, Dancey J, Arbuck S, Gwyther S, Mooney M, et al: New response evaluation criteria in solid tumours: Revised RECIST guideline (version 1.1). Eur J Cancer 45: 228-247, 2009.

21. Schuierer S, Carbone W, Knehr J, Petitjean V, Fernandez A, Sultan M and Roma G: A comprehensive assessment of RNA-seq protocols for degraded and low-quantity samples. BMC Genomics 18: 1-13,2017.

22. Andrews S: FastQC: A quality control tool for high throughput sequence data. Babraham Institute, Cambridge, 2010 http://www.bioinformatics.babraham.ac.uk/projects/fastqc/. Accessed November 11, 2019.

23. Krueger F: Trim galore: A wrapper tool around Cutadapt and FastQC to consistently apply quality and adapter trimming to FastQ files, with some extra functionality for MspI-digested RRBS-type (Reduced Representation Bisufite-Seq) libraries. Babraham Institute, Cambridge, 2015. https://www.bioinformatics.babraham.ac.uk/projects/trim_galore/. Accessed November 11, 2019

24. Dobin A, Davis CA, Schlesinger F, Drenkow J, Zaleski C, Jha S Batut P, Chaisson M and Gingeras TR: STAR: Ultrafast universal RNA-seq aligner. Bioinformatics 29: 15-21, 2013.

25. Li B and Dewey CN: RSEM: Accurate transcript quantification from RNA-Seq data with or without a reference genome. BMC Bioinformatics 12: 323, 2011 .
26. Love MI, Huber W and Anders S: Moderated estimation of fold change and dispersion for RNA-seq data with DESeq2. Genome Biol 15: 550, 2014

27. Yu G, Wang LG, Han Y and He QY: clusterProfiler: An R package for comparing biological themes among gene clusters. OMICS 16: 284-287, 2012

28. Kanehisa M, Sato Y, Kawashima M, Furumichi M and Tanabe M: KEGG as a reference resource for gene and protein annotation. Nucleic Acids Res 44: D457-D462, 2015.

29. Kim JE, Chun SM, Hong YS, Kim KP, Kim SY, Kim J, Sung CO, Cho EJ, Kim TW and Jang SJ: Mutation burden and I index for detection of microsatellite instability in colorectal cancer by targeted next-generation sequencing. J Mol Diagn 21: 241-250, 2018.

30. Philpott C, Tovell H, Frayling IM, Cooper DN and Upadhyaya M: The NF1 somatic mutational landscape in sporadic human cancers. Hum Genomics 11: 13, 2017.

31. Redig AJ, Capelletti M, Dahlberg SE, Sholl LM, Mach S, Fontes C, Shi Y, Chalasani P and Jänne PA: Clinical and molecular characteristics of NF1-mutant lung cancer. Clin Cancer Res 22: 3148-3156, 2016.

32. Hayashi T, Desmeules P, Smith RS, Drilon A, Somwar R and Ladanyi M: RASA1 and NF1 are preferentially co-mutated and define a distinct genetic subset of smoking-associated non-small cell lung carcinomas sensitive to MEK inhibition. Clin Cancer Res 24: 1436-1447, 2018.

33. Dischinger PS, Tovar EA, Essenburg CJ, Madaj ZB, Gardner EE, Callaghan ME, Turner AN, Challa AK, Kempston T, Eagleson B, et al: NF1 deficiency correlates with estrogen receptor signaling and diminished survival in breast cancer. NPJ Breast Cancer 4: 29, 2018

34. Pearson A, Proszek P, Pascual J, Fribbens C, Shamsher MK, Kingston B, O'Leary B, Herrera-Abreu MT, Cutts RJ, Garcia-Murillas I, et al: Inactivating NF1 mutations are enriched in advanced breast cancer and contribute to endocrine therapy resistance. Clin Cancer Res 26: 608-622, 2020.

35. Mei Z, Shao YW, Lin P, Cai X, Wang B, Ding Y, Ma X, Wu X, Xia Y, Zhu D, et al: SMAD4 and NF1 mutations as potential biomarkers for poor prognosis to cetuximab-based therapy in Chinese metastatic colorectal cancer patients. BMC Cancer 18: 479, 2018.

36. Cancer Genome Atlas Network: Comprehensive molecular characterization of human colon and rectal cancer. Nature 487: 330-337, 2012.

37. Meng M, Zhong K, Jiang T, Liu Z, Kwan HY and Su T: The current understanding on the impact of KRAS on colorectal cancer. Biomed Pharmacother 140: 111717, 2021.

38. McFall T, Diedrich JK, Mengistu M,Littlechild SL, Paskvan KV, Sisk-Hackworth L, Moresco JJ, Shaw AS and Stites EC: A systems mechanism for KRAS mutant allele-specific responses to targeted therapy. Sci Signal 12: eaaw8288, 2019.

39. Georgiou A, Stewart A, Cunningham D, Banerji U and Whittaker SR: Inactivation of NF1 promotes resistance to EGFR inhibition in KRAS/NRAS/BRAF ${ }^{\mathrm{V} 600}$-wild-type colorectal cancer. Mol Cancer Res 18: 835-846, 2020.

40. Bray SM, Lee J, Kim ST, Hur JY, Ebert PJ, Calley JN, Wulur IH, Gopalappa T, Wong SS, Qian HR, et al: Genomic characterization of intrinsic and acquired resistance to cetuximab in colorectal cancer patients. Sci Rep 9: 15365, 2019.

41. AACR Project GENIE: Powering precision medicine through an international consortium. Cancer Discov 7: 818-831, 2017.

42. Kandoth C, McLellan MD, Vandin F, Ye K, Niu B, Lu C, Xie M, Zhang Q, McMichael JF, Wyczalkowski MA, et al: Mutational landscape and significance across 12 major cancer types. Nature 502: 333-339, 2013.

43. Baeissa HM, Benstead-Hume G, Richardson CJ and Pearl FM: Mutational patterns in oncogenes and tumour suppressors. Biochem Soc Trans 44: 925-931, 2016.

44. Gutmann DH, Wu YL, Hedrick NM, Zhu Y, Guha A and Parada LF: Heterozygosity for the neurofibromatosis 1 (NF1) tumor suppressor results in abnormalities in cell attachment, spreading and motility in astrocytes. Hum Mol Genet 10: 3009-3016, 2001.

45. Brosseau JP, Liao CP, Wang Y, Ramani V, Vandergriff T, Lee M, Patel A and Ariizumi K: NF1 heterozygosity fosters de novo tumorigenesis but impairs malignant transformation. Nat Commun 9: 5014, 2018.

46. Guo XE, Ngo B, Modrek AS and Lee WH: Targeting tumor suppressor networks for cancer therapeutics. Curr Drug Targets 15: 2-16, 2014. 
47. Bai RY, Esposito D, Tam AJ, McCormick F, Riggins GJ, Clapp DW and Staedtke V: Feasibility of using NF1-GRD and AAV for gene replacement therapy in NF1-associated tumors. Gene Ther 26: 277-286, 2019.

48. Kong N, Tao W, Ling X, Wang J, Xiao Y, Shi S, Ji X, Shajii A, Gan ST, Kim NY, et al: Synthetic mRNA nanoparticle-mediated restoration of p53 tumor suppressor sensitizes p53-deficient cancers to mTOR inhibition. Sci Transl Med 11: eaaw1565, 2019

49. El Sharkawi FZ, Ewais SM, Fahmy RH and Rashed LA: PTEN and TRAIL genes loaded zein nanoparticles as potential therapy for hepatocellular carcinoma. J Drug Target 25: 513-522, 2017.
50. Groelz D, Sobin L, Branton P, Compton C, Wyrich R and Rainen L: Non-formalin fixative versus formalin-fixed tissue: A comparison of histology and RNA quality. Exp Mol Pathol 94: 188-194, 2013.

(c) (i) $($ This work is licensed under a Creative Commons Attribution-NonCommercial-NoDerivatives 4.0 International (CC BY-NC-ND 4.0) License. 